\title{
Existence and continuous dependence for weighted fractional differential equations with infinite delay
}

\section{Qixiang Dong*}

"Correspondence:

qxdongyz@outlook.com; qxdong@yzu.edu.cn

School of Mathematical Sciences, Yangzhou University, Yangzhou,

225002, P.R. China

\begin{abstract}
This paper is concerned with weighted fractional differential equations with infinite delay, the Riemann-Liouville derivative, and nonzero initial values. Existence and continuous dependence results of solutions are obtained. An illustrative example is also presented.
\end{abstract}

Keywords: fractional integral; fractional derivative; functional differential equation; infinite delay

\section{Introduction}

Recently, fractional differential equations have attracted a considerable interest in both mathematics and applications, since they have been proved to be valuable tools in modeling many physical phenomena. There has been a significant development in fractional differential equations in the past decades [1-12]. Among these works, some authors studied functional fractional differential equations [5-8, 10]. For example, in [5], Benchohra et al. studied the fractional order differential equations

$$
D^{\alpha} y(t)=f\left(t, y_{t}\right), \quad t \in[0, b], 0<\alpha<1
$$

with infinite delay

$$
y(t)=\phi(t), \quad t \in(-\infty, 0],
$$

where $D^{\alpha}$ is the standard Riemann-Liouville fractional derivative, $\phi \in \mathcal{B}$, the phase space defined axiomatically by Hale and Kato, and $y_{t}(\theta)=y(t+\theta)$ for $\theta<0$. Some existence results are obtained in the special case $y(0)=0$. Henderson and Ouahab also studied the multivalued version of (1.1) (i.e., fractional differential inclusion) with finite delay in [6]. Zhang [7] discussed the linear fractional order time-delay system

$$
\begin{aligned}
& D^{\alpha} x(t)=A_{0} x(t)+A_{1} x(t-r)+f(t), \quad t \geq 0,0<\alpha<1, \\
& x(t)=\phi(t), \quad t \in[-r, 0]
\end{aligned}
$$

(2014 Dong; licensee Springer. This is an Open Access article distributed under the terms of the Creative Commons Attribution License (http://creativecommons.org/licenses/by/2.0), which permits unrestricted use, distribution, and reproduction in any medium, provided the original work is properly cited. 
in a finite dimensional space $\mathbf{R}^{n}$ and got some existence and stability results. In [8], Lakshmikantham concerned some basic theory for functional fractional differential equations.

However, it is known that the Riemann-Liouville fractional derivative of a function $y$ is unbounded at some neighborhoods of the initial point 0 , except that $y(0)=0$. For this reason, when $y(0) \neq 0$, the solutions to the functional fractional differential equations given in the mentioned papers may not be well defined.

We investigate some examples. Firstly, we consider fractional differential equations with infinite delay. Let $C^{0}=\left\{\phi \in C((-\infty, 0] ; \mathbf{R}): \phi\right.$ is bounded on $(-\infty, 0]$, and $\lim _{\theta \rightarrow-\infty} \phi(\theta)=$ 0 \}. Then $C^{0}$ satisfies axioms (A1), (A2) and (B) (specified later, also see [13]), with $H=1$ and $K(t)=M(t)=1$ for $t \geq 0$ [13]. Let $\phi(\theta)=e^{\theta}$ for $\theta \in(-\infty, 0]$ in (1.2). Obviously, $\phi \in C^{0}$. We take $\alpha=\frac{1}{4}$ and $f\left(t, y_{t}\right)=y(t-b)$. Then equations (1.1)-(1.2) become

$$
\begin{aligned}
& D^{1 / 4} y(t)=y(t-b), \quad t \in(0, b], \\
& y(t)=e^{t}, \quad t \in(-\infty, 0] .
\end{aligned}
$$

Then we get that $y(t-b)=e^{t-b}$ for $t \in(0, b]$. Since $I^{\alpha} D^{\alpha} y(t)=y(t)+C t^{\alpha-1}$ for some constant $C$, we can apply the fractional integral operator $I^{\frac{1}{4}}$ to both sides of (1.5). A direct computation gives

$$
\begin{aligned}
y(t) & =I^{1 / 4}\left(e^{t-b}\right)+C t^{-3 / 4} \\
& =e^{-b} \cdot I^{1 / 4}\left(\sum_{k=0}^{\infty} \frac{t^{k}}{k !}\right)+C t^{-3 / 4} \\
& =e^{-b} \cdot \sum_{k=0}^{\infty} \frac{\Gamma(k+1) t^{k+1 / 4}}{k ! \Gamma(k+1+1 / 4)}+C t^{-3 / 4} \\
& =e^{-b} \cdot t^{1 / 4} \sum_{k=0}^{\infty} \frac{t^{k}}{\Gamma(k+5 / 4)}+C t^{-3 / 4}
\end{aligned}
$$

for $t \in[0, b]$ and some constant $C$, which is unbounded at any right neighborhood of 0 . Consequently, equations (1.5)-(1.6) cannot have any continuous solution on $(-\infty, b]$.

Similarly, for the case of finite delay, we consider the following fractional differential equation on $\mathbf{R}$ with finite delay:

$$
\begin{aligned}
& D^{1 / 2} y(t)=y(t-r), \quad t \in(0, b], \\
& y(t)=1, \quad t \in[-r, 0] .
\end{aligned}
$$

Then $y(t-r)=1$ for $t \in(0, r]$, and equation (1.8) reduces to $D^{1 / 2} y(t)=1$ for $t \in(0, r]$. Applying the fractional integral operator $I^{1 / 2}$ to both sides, we get that

$$
y(t)=\frac{1}{2 \sqrt{\pi}} t^{1 / 2}+C t^{-1 / 2}
$$

for $t \in[0, r]$, which is still unbounded in the right neighborhood of 0 .

From the above examples we can see that, in the case of nonzero initial value, functional fractional differential equations with Riemann-Liouville derivative and either finite or infinite delay may not have 'classical' solutions. Moreover, the functions $y_{t}$ that appeared in 
these equations, defined by $y_{t}(\theta)=y(t+\theta)$ for $\theta \leq 0$, may not be well defined, or not in the phase space. On the other hand, the Riemann-Liouville fractional derivative of the constant function is not zero. In fact, if $h(t)=C \neq 0$ for $t \in[a, b]$ and $0<\alpha<1$, then $D_{a}^{\alpha} h(t)=C \frac{(t-a)^{-\alpha}}{\Gamma(1-\alpha)} \neq 0$. Hence the nonzero initial value problems cannot be transformed to zero initial value problem by the parallel shift method.

Motivated by the above comment, and inspired by [2] and [3], in this paper, we consider the weighted functional fractional differential equation with infinite delay of the form

$$
\begin{aligned}
& D^{\alpha} y(t)=f\left(t, \tilde{y}_{t}\right), \quad t \in(0, b], \\
& \tilde{y}_{0}=\phi \in \mathcal{B},
\end{aligned}
$$

where $0<\alpha \leq 1, D^{\alpha}$ is the Riemann-Liouville fractional derivative, $\tilde{y}(t)=t^{1-\alpha} y(t), f$ : $(0, b] \times \mathcal{B} \rightarrow \mathbf{R}$ is a given function satisfying some assumptions, and $\mathcal{B}$ is the phase space that will be specified later. We give the definition of solutions and investigate the existence and continuous dependence of solutions to such equations in the space $C_{1-\alpha}((a, b])$. An example is presented to illustrate the results.

\section{Preliminaries and lemmas}

In this section we collect some definitions and results needed in our further investigations.

Let us denote by $C((a, b])$ the space of all continuous real functions defined on $(a, b]$ and by $L_{\text {loc }}^{1}(a, b)$ the space of all real functions defined on $(a, b)$ which are locally Lebesgue integrable. We also consider the space $C_{r}((a, b])$ consisting of all continuous functions $f:(a, b] \rightarrow \mathbf{R}$ such that $\lim _{t \rightarrow a}(t-a)^{r} f(t)$ exists, with the norm $\|f\|_{C_{r}}=\sup \left\{\left|(t-a)^{r} f(t)\right| ; t \in\right.$ $(a, b]\}$.

Definition 2.1 [1] Let $\alpha>0$ be a fixed number. The Riemann-Liouville fractional integral of order $\alpha>0$ of the function $h:[a, b] \rightarrow \mathbf{R}$ is defined by

$$
I_{a}^{\alpha} h(t)=\frac{1}{\Gamma(\alpha)} \int_{a}^{t}(t-s)^{\alpha-1} h(s) d s, \quad t \in[a, b]
$$

provided the right-hand side is pointwisely defined, where $\Gamma(\cdot)$ denotes the well-known gamma function, i.e., $\Gamma(z)=\int_{0}^{\infty} \mathrm{e}^{-t} t^{z-1} d t$.

Definition 2.2 [1] Let $\alpha>0$ be fixed and $n=[\alpha]+1$. The Riemann-Liouville fractional derivative of order $\alpha$ of $h:(a, b] \rightarrow \mathbf{R}$ at the point $t$ is defined by

$$
D_{a}^{\alpha} h(t)=\frac{1}{\Gamma(n-\alpha)} \frac{d^{n}}{d t^{n}} \int_{a}^{t}(t-s)^{n-\alpha-1} h(s) d s, \quad t \in[a, b]
$$

provided the right-hand side is pointwisely defined, where $[\alpha]$ denotes the integer part of the real number $\alpha$.

When $0<\alpha<1$, then

$$
D_{a}^{\alpha} h(t)=\frac{1}{\Gamma(1-\alpha)} \frac{d}{d t} \int_{a}^{t}(t-s)^{\alpha-1} h(s) d s .
$$

For simplicity, when $a=0$, we denote $D_{0}^{\alpha}$ and $I_{0}^{\alpha}$ by $D^{\alpha}$ and $I^{\alpha}$, respectively. 
Lemma 2.3 [3] Let $0<\alpha<1$. Then the unique solutions to the equation $D^{\alpha} h(t)=0$ are given by the formula

$$
h(t)=C t^{\alpha-1},
$$

for $t>0$, where $C \in \mathbf{R}$ is a constant, provided $h \in C((0, b]) \cap L_{\mathrm{loc}}^{1}(0, b)$. Further, if $f \in$ $C((0, b]) \cap L_{\mathrm{loc}}^{1}(a, b)$ such that $D^{\alpha} f \in C((0, b]) \cap L_{\mathrm{loc}}^{1}(0, b)$, then

$$
I^{\alpha} D^{\alpha} f(t)=f(t)+C t^{\alpha-1}
$$

for $t>0$ and some constant $C \in \mathbf{R}$.

In the literature devoted to equations with infinite delay, the selection of the state space $\mathcal{B}$ plays an important role in the study of both qualitative and quantitative theory. A usual choice is a semi-normed space satisfying suitable axioms, which was introduced by Hale and Kato [14]. For a detailed discussion on the topic, we refer to the book by Hino et al. [13].

Definition 2.4 [13] A linear topological space of functions from $(-\infty, 0]$ into $X$, with seminorm $\|\cdot\|_{\mathcal{B}}$, is called an admissible phase space if $\mathcal{B}$ has the following properties.

(A1) There exist a positive constant $H$ and functions $K(\cdot), M(\cdot):[0,+\infty) \rightarrow[0,+\infty)$, with $K$ continuous and $M$ locally bounded, such that for any $a, b \in \mathbf{R}$ and $b>a$, if $x:(-\infty, b] \rightarrow X, x_{a} \in \mathcal{B}$, and $x(\cdot)$ is continuous on $[a, b]$, then for every $t \in[a, b]$, the following conditions hold:

(i) $x_{t} \in \mathcal{B}$;

(ii) $\|x(t)\| \leq H\left\|x_{t}\right\|_{\mathcal{B}}$ for some $H>0$;

(iii) $\left\|x_{t}\right\|_{\mathcal{B}} \leq K(t-a) \sup _{a \leq s \leq t}\|x(s)\|+M(t-a)\left\|x_{a}\right\|_{\mathcal{B}}$.

(A2) For the function $x(\cdot)$ in (A1), $t \mapsto x_{t}$ is a $\mathcal{B}$-valued continuous function for $t \in[a, b]$.

(B) The space $\mathcal{B}$ is complete.

\section{Existence results}

We begin with the definition of solutions to the weighted functional fractional differential equations.

Definition 3.1 A function $y:(-\infty, b] \rightarrow \mathbf{R}$ is said to be a solution to (1.9)-(1.10), if $\left.y\right|_{(0, b]} \in$ $C((0, b]) \cap L_{\text {loc }}^{1}(0, b), \tilde{y}_{0}=\phi$ and satisfies (1.9).

Example 3.1 Consider the weighted version of the previous example

$$
\begin{aligned}
& D^{1 / 4} y(t)=\tilde{y}(t-b), \quad t \in(0, b], \\
& \tilde{y}(t)=e^{t}, \quad t \in(-\infty, 0] .
\end{aligned}
$$

By computation we also get that for $t \in(0, b]$,

$$
y(t)=e^{-b} \cdot t^{1 / 4} \sum_{k=0}^{\infty} \frac{t^{k}}{\Gamma(k+5 / 4)}+C t^{-3 / 4}=e^{-b} \cdot t^{1 / 4} E_{1,5 / 4}(t)+C t^{-3 / 4} .
$$


Then $\tilde{y}(t)=e^{-b} \cdot t^{1} E_{1,5 / 4}(t)+C$. Since $\tilde{y}(0)=1$, we get that $C=1$. Therefore, the solution to (3.1)-(3.2) is

$$
y(t)= \begin{cases}e^{-b} \cdot t^{1 / 4} E_{1,5 / 4}(t)+t^{-3 / 4}, & t \in(0, b], \\ e^{t}, & t \in(-\infty, 0],\end{cases}
$$

where $E_{\alpha, \beta}(\cdot)$ is the Mittag-Leffler function, i.e., $E_{\alpha, \beta}(t)=\sum_{k=0}^{\infty} \frac{t^{k}}{\Gamma(\alpha k+\beta)}$. Note that

$$
\tilde{y}(t)=t^{3 / 4} y(t)= \begin{cases}e^{-b} \cdot t E_{1,5 / 4}(t)+1, & t \in(0, b] \\ e^{t}, & t \in(-\infty, 0]\end{cases}
$$

is continuous on $(-\infty, b]$, and therefore, $\tilde{y}_{t}$ is well defined for $t \in[0, b]$.

For the existence results on problem (1.9)-(1.10), we need to transform the fractional differential equation into an integral equation. From Lemma 2.3 we can obtain that if $0<$ $\alpha<1$ and $h \in C((0, b]) \cap L_{\mathrm{loc}}^{1}(0, b)$, then the function $y$ solves the fractional differential equation

$$
D^{\alpha} y(t)=h(t), \quad t \in(0, b]
$$

if and only if $y$ satisfies

$$
y(t)=\frac{1}{\Gamma(\alpha)} \int_{0}^{t}(t-s)^{\alpha-1} h(s) d s+C t^{\alpha-1}, \quad t \in(0, b]
$$

for some constant $C$ [3].

We first give an existence result based on the Banach contraction principle. We list the hypotheses.

$\left(\mathrm{H}_{1}\right) f:(0, b] \times \mathcal{B} \rightarrow \mathbf{R}$ is continuous.

$\left(\mathrm{H}_{2}\right)$ There exists a constant $L>0$ such that

$$
|f(t, u)-f(t, v)| \leq L\|u-v\|_{\mathcal{B}}
$$

for $t \in(0, b]$ and every $u, v \in \mathcal{B}$.

Theorem 3.2 Assume that $\left(\mathrm{H}_{1}\right)$ and $\left(\mathrm{H}_{2}\right)$ hold. Then there exists a unique solution to (1.9)(1.10) on $(-\infty, b]$.

Proof By Lemma 2.3 and the above remark, a function $y$ is a solution to (1.9)-(1.10) if and only if $y$ satisfies

$$
y(t)= \begin{cases}\frac{1}{\Gamma(\alpha)} \int_{0}^{t}(t-s)^{\alpha-1} f\left(s, \tilde{y}_{s}\right) d s+\phi(0) t^{\alpha-1}, & t \in(0, b], \\ \phi(t), & t \in(-\infty, 0] .\end{cases}
$$

For given $\phi:(-\infty, 0]$ which belongs to $\mathcal{B}$, let $\tilde{\phi}$ be a function defined by

$$
\tilde{\phi}(t)= \begin{cases}0, & t \in(0, b] \\ \phi(t), & t \in(-\infty, 0] .\end{cases}
$$


Then we have $\tilde{\phi}_{0}=\phi$. For $z \in C_{1-\alpha}((0, b] ; \mathbf{R})$, where $C_{1-\alpha}((0, b] ; \mathbf{R})$ is the Banach space consisting of all continuous functions $f:(0, b] \rightarrow \mathbf{R}$ such that $\lim _{t \rightarrow 0} t^{1-\alpha} f(t)$ exists, endowed with the norm $\|f\|_{C_{1-\alpha}}=\sup \left\{\left|t^{1-\alpha} f(t)\right| ; t \in(0, b]\right\}$, we extend $\tilde{z}$ to $(-\infty, b]$, also denoted by $\tilde{z}$, defined by

$$
\tilde{z}(t)= \begin{cases}t^{1-\alpha} z(t), & t \in(0, b], \\ 0, & t \in(-\infty, 0] .\end{cases}
$$

It is easily seen that if $y(\cdot)$ satisfies the integral equation

$$
y(t)=\frac{1}{\Gamma(\alpha)} \int_{0}^{t}(t-s)^{\alpha-1} f\left(s, \tilde{y}_{s}\right) d s+\phi(0) t^{\alpha-1}, \quad t>0,
$$

we can decompose $y(\cdot)$ as $y(t)=\phi(t)+z(t)$, which implies that $\tilde{y}_{t}=\tilde{\phi}_{t}+\tilde{z}_{t}$ for $t \in(0, b]$, and the function $z(\cdot)$ satisfies

$$
z(t)=\frac{1}{\Gamma(\alpha)} \int_{0}^{t}(t-s)^{\alpha-1} f\left(s, \tilde{\phi}_{s}+\tilde{z}_{s}\right) d s+\phi(0) t^{\alpha-1}, \quad t>0
$$

Set $W=\left\{z:(-\infty, b] \rightarrow \mathbf{R} ;\left.z\right|_{(0, b]} \in C_{1-\alpha}((0, b] ; \mathbf{R}), z_{0}=0\right\}$. For $z \in W$, define $\|z\|_{W}=$ $\left\|z_{0}\right\|_{\mathcal{B}}+\|z\|_{C_{1-\alpha}}=\|z\|_{C_{1-\alpha}}$, then $\left(W,\|z\|_{W}\right)$ becomes a Banach space. Define an operator $P: W \rightarrow W$ by

$$
(P z)(t)=\frac{1}{\Gamma(\alpha)} \int_{0}^{t}(t-s)^{\alpha-1} f\left(s, \tilde{\phi}_{s}+\tilde{z}_{s}\right) d s+\phi(0) t^{\alpha-1}, \quad t>0
$$

We can see that if $z \in W$ is a fixed point of $P$, then $y=z+\tilde{\phi}$ is a solution of (1.9)-(1.10).

Let $K_{b}=\sup \{K(t) ; t \in[0, b]\}$, where $K(\cdot)$ is the function that appeared in Definition 2.4. Let $N=\left[b\left(2 L K_{b} b^{1-\alpha} / \Gamma(1+\alpha)\right)^{1 / \alpha}\right]$, and $h_{i}=i b / N$. Then $0=h_{1}<h_{2}<\cdots<h_{N}=b$ and

$$
\frac{L K_{b} b^{1-\alpha}\left(h_{i+1}-h_{i}\right)^{\alpha}}{\Gamma(1+\alpha)}<\frac{1}{2}
$$

for $i=1,2, \ldots, N$.

We first focus on the interval $\left(0, h_{1}\right]$. Let $W_{1}=\left\{z:\left(-\infty, h_{1}\right] \rightarrow \mathbf{R} ;\left.z\right|_{\left(0, h_{1}\right]} \in C_{1-\alpha}\left(\left(0, h_{1}\right] ; \mathbf{R}\right)\right.$, $\left.\left.z\right|_{0}=0\right\}$ and define $\|z\|_{W 1}=\left\|z_{0}\right\|_{\mathcal{B}}+\sup \left\{\left|t^{1-\alpha} z(t)\right| ; 0<t \leq h_{1}\right\}=\sup \left\{\left|t^{1-\alpha} z(t)\right| ; 0<t \leq h_{1}\right\}$ for $z \in W_{1}$. Then $\left(W_{1},\|z\|_{W 1}\right)$ is a Banach space. Define the operator $P_{1}: W_{1} \rightarrow W_{1}$ by

$$
\left(P_{1} z\right)(t)=\frac{1}{\Gamma(\alpha)} \int_{0}^{t}(t-s)^{\alpha-1} f\left(s, \tilde{\phi}_{s}+\tilde{z}_{s}\right) d s+\phi(0) t^{\alpha-1}, \quad t \in\left(0, h_{1}\right] .
$$

For $z, z^{*} \in W_{1}$ and $t \in\left(0, h_{1}\right]$, we have

$$
\begin{aligned}
& \left|t^{1-\alpha}\left(P_{1} z\right)(t)-t^{1-\alpha}\left(P_{1} z^{*}\right)(t)\right| \\
& \quad \leq \frac{t^{1-\alpha}}{\Gamma(\alpha)} \int_{0}^{t}(t-s)^{\alpha-1}\left|f\left(s, \tilde{\phi}_{s}+\tilde{z}_{s}\right)-f\left(s, \tilde{\phi}_{s}+\tilde{z}_{s}^{*}\right)\right| d s \\
& \quad \leq \frac{L t^{1-\alpha}}{\Gamma(\alpha)} \int_{0}^{t}(t-s)^{\alpha-1}\left\|\tilde{z}_{s}-\tilde{z}_{s}^{*}\right\|_{\mathcal{B}} d s .
\end{aligned}
$$


Since

$$
\begin{aligned}
& \left\|\tilde{z}_{s}-\tilde{z}_{s}^{*}\right\|_{\mathcal{B}} \\
& \quad \leq K(s) \sup _{0 \leq \tau \leq s}\left\{\left|\tilde{z}(\tau)-\tilde{z}^{*}(\tau)\right|\right\}+M(s)\left\|\tilde{z}_{0}-\tilde{z}_{0}^{*}\right\|_{\mathcal{B}} \\
& \quad \leq K_{b} \sup _{0 \leq \tau \leq s}\left\{\left|\tau^{1-\alpha} z(\tau)-\tau^{1-\alpha} z^{*}(\tau)\right|\right\} \\
& \quad \leq K_{b}\left\|z-z^{*}\right\|_{W 1},
\end{aligned}
$$

we have

$$
\begin{aligned}
& \left|t^{1-\alpha}\left(P_{1} z\right)(t)-t^{1-\alpha}\left(P_{1} z^{*}\right)(t)\right| \\
& \quad \leq \frac{L K_{b} t^{1-\alpha}}{\Gamma(\alpha)} \int_{0}^{t}(t-s)^{\alpha-1} d s\left\|z-z^{*}\right\|_{W 1} \\
& \leq \frac{L K_{b} b^{1-\alpha} h_{1}^{\alpha}}{\Gamma(1+\alpha)}\left\|z-z^{*}\right\|_{W 1},
\end{aligned}
$$

and hence

$$
\left\|P_{1} z-P_{1} z^{*}\right\|_{W 1} \leq \frac{L K_{b} b^{1-\alpha} h_{1}^{\alpha}}{\Gamma(1+\alpha)}\left\|z-z^{*}\right\|_{W 1}
$$

From (3.4) and the Banach contraction principle we know that there exists a unique $z \in W_{1}$ satisfying

$$
z(t)=\frac{1}{\Gamma(\alpha)} \int_{0}^{t}(t-s)^{\alpha-1} f\left(s, \tilde{\phi}_{s}+\tilde{z}_{s}\right) d s+\phi(0) t^{\alpha-1}
$$

for $t \in\left(0, h_{1}\right]$, which is the unique solution to the integral equation (3.3) on the interval $\left(0, h_{1}\right]$.

Next we consider the interval $\left[h_{1}, h_{2}\right]$. Restrict the functions $z \in W$ on the interval $\left[h_{1}, h_{2}\right]$ to construct $W_{2}$ and define $\|z\|_{W 2}=\left\|z_{0}\right\|_{\mathcal{B}}+\sup \left\{\left|t^{1-\alpha} z(t)\right| ; h_{1}<t \leq h_{2}\right\}=\sup \left\{\left|t^{1-\alpha} z(t)\right| ; h_{1}<\right.$ $\left.t \leq h_{2}\right\}$ for $z \in W_{2}$. Then $\left(W_{2},\|z\|_{W 2}\right)$ is a Banach space. For $t \in\left[h_{1}, h_{2}\right]$, rewrite equation (3.3) as

$$
\begin{aligned}
z(t)= & \frac{1}{\Gamma(\alpha)} \int_{h_{1}}^{t}(t-s)^{\alpha-1} f\left(s, \tilde{\phi}_{s}+\tilde{z}_{s}\right) d s \\
& +\frac{1}{\Gamma(\alpha)} \int_{0}^{h_{1}}(t-s)^{\alpha-1} f\left(s, \tilde{\phi}_{s}+\tilde{z}_{s}\right) d s+\phi(0) t^{\alpha-1}
\end{aligned}
$$

Since the function $z$ is uniquely defined on $\left(0, h_{1}\right]$, the second integral can be considered as a known function. Using the same arguments as above, we can obtain that there exists a unique function $z \in W_{2}$ satisfying

$$
\begin{aligned}
z(t)= & \frac{1}{\Gamma(\alpha)} \int_{h_{1}}^{t}(t-s)^{\alpha-1} f\left(s, \tilde{\phi}_{s}+\tilde{z}_{s}\right) d s \\
& +\frac{1}{\Gamma(\alpha)} \int_{0}^{h_{1}}(t-s)^{\alpha-1} f\left(s, \tilde{\phi}_{s}+\tilde{z}_{s}\right) d s+\phi(0) t^{\alpha-1}
\end{aligned}
$$


for $t \in\left[h_{1}, h_{2}\right]$, which is the unique solution to the integral equation (3.3) on the interval $\left[h_{1}, h_{2}\right]$. Taking the next interval $\left[h_{2}, h_{3}\right]$, repeating this process, we conclude that there exists a unique solution to the integral equation (3.3) on the interval $\left(0, h_{N}\right]=(0, b]$. Set $y=z+\tilde{\phi}$, then $y$ is the unique solution to the fractional differential equation (1.9)-(1.10).

Below we consider the existence result which is based on the Schauder fixed point theorem. We need the following hypothesis.

$\left(\mathrm{H}_{3}\right)$ There exist an $\eta \in L^{p}(0, b)$ with $p>1 / \alpha$ and a continuously non-decreasing function $\Omega:[0,+\infty) \rightarrow[0,+\infty)$ such that

$$
|f(t, u)| \leq \eta(t) \Omega\left(\|u\|_{\mathcal{B}}\right)
$$

for $t \in(0, b]$ and every $u \in \mathcal{B}$.

Theorem 3.3 Assume that hypotheses $\left(\mathrm{H}_{1}\right)$ and $\left(\mathrm{H}_{3}\right)$ hold. If

$$
\limsup _{r \rightarrow+\infty} \frac{\Omega(r)}{r}<\frac{\Gamma(1+\alpha)}{K_{b} b^{2}\|\eta\|_{p}}
$$

then there exists at least a solution to (1.9)-(1.10) on $(-\infty, b]$.

Proof As in the proof of Theorem 3.2, we define the operator $P: W \rightarrow W$. The continuity of $P$ can be derived by hypothesis $\left(\mathrm{H}_{3}\right)$ and the Lebesgue dominated convergence theorem. We will verify that $P$ is completely continuous.

We first show that $P$ maps bounded subsets in $W$ into bounded subsets. Let $B_{r}=\{z \in$ $\left.W ;\|z\|_{W} \leq r\right\}$. Then, for any $z \in B_{r}$ and $t \in(0, b]$, we have

$$
\begin{aligned}
\left|t^{1-\alpha}(P z)(t)\right| & \leq \frac{t^{1-\alpha}}{\Gamma(\alpha)} \int_{0}^{t}(t-s)^{\alpha-1}\left|f\left(s, \tilde{\phi}_{s}+\tilde{z}_{s}\right)\right| d s+|\phi(0)| \\
& \leq \frac{b^{1-\alpha}}{\Gamma(\alpha)} \int_{0}^{t}(t-s)^{\alpha-1} \eta(s) \Omega\left(\left\|\tilde{\phi}_{s}+\tilde{z}_{s}\right\|_{\mathcal{B}}\right) d s+|\phi(0)| .
\end{aligned}
$$

Since

$$
\begin{aligned}
\left\|\tilde{z}_{s}-\tilde{\phi}_{s}\right\|_{\mathcal{B}} & \leq K(s) \sup _{0 \leq \tau \leq s}|\tilde{z}(\tau)|+M(s)\left\|\tilde{z}_{0}\right\|_{\mathcal{B}}+K(s) \sup _{0 \leq \tau \leq s}|\tilde{\phi}(\tau)|+M(s)\left\|\tilde{\phi}_{0}\right\|_{\mathcal{B}} \\
& \leq K_{b} r+M_{b}\|\phi\|_{\mathcal{B}}
\end{aligned}
$$

where $M_{b}=\sup _{0 \leq s \leq b} M(s)$, it follows from $\left(\mathrm{H}_{3}\right)$ and Holder's inequality that

$$
\begin{aligned}
\left|t^{1-\alpha}(P z)(t)\right| & \leq \frac{b^{1-\alpha}}{\Gamma(\alpha)} \int_{0}^{t}(t-s)^{\alpha-1} \eta(s) d s \Omega\left(K_{b} r+M_{b}\|\phi\|_{\mathcal{B}}\right)+|\phi(0)| \\
& \leq \frac{b^{1-\alpha}}{\Gamma(\alpha)} \Omega\left(K_{b} r+M_{b}\|\phi\|_{\mathcal{B}}\right)\left(\int_{0}^{t}(t-s)^{(\alpha-1) q} d s\right)^{1 / q}\|\eta\|_{p}+|\phi(0)| \\
& \leq \Omega\left(K_{b} r+M_{b}\|\phi\|_{\mathcal{B}}\right) \frac{b^{2}\|\eta\|_{p}}{\Gamma(1+\alpha)}+|\phi(0)|:=l,
\end{aligned}
$$

where $\|\eta\|_{p}=\int_{0}^{b}|\eta(s)| d s$ and $q>1$ with $1 / p+1 / q=1$. Therefore, $\|P z\|_{W} \leq l$ for every $z \in B_{r}$, which implies that $P$ maps bounded subsets into bounded subsets in $W$. 
Next, we prove that $P$ maps bounded subsets into equicontinuous subsets in $W$. Let $z \in B_{r}$ and $t_{1}, t_{2} \in(0, b]$ with $t_{1}<t_{2}$, we have

$$
\begin{aligned}
\left|t_{2}^{1-\alpha}(P z)\left(t_{2}\right)-t_{1}^{1-\alpha}(P z)\left(t_{1}\right)\right| & \\
\leq & \frac{t_{2}^{1-\alpha}-t_{1}^{1-\alpha}}{\Gamma(\alpha)} \int_{0}^{t_{2}}\left(t_{2}-s\right)^{\alpha-1}\left|f\left(s, \tilde{\phi}_{s}+\tilde{z}_{s}\right)\right| d s \\
& +\frac{t_{1}^{1-\alpha}}{\Gamma(\alpha)}\left[\int_{0}^{t_{1}}\left|\left(t_{2}-s\right)^{\alpha-1}-\left(t_{1}-s\right)^{\alpha-1}\right|\left|f\left(s, \tilde{\phi}_{s}+\tilde{z}_{s}\right)\right| d s\right. \\
& \left.+\int_{t_{1}}^{t_{2}}\left(t^{2}-s\right)^{\alpha-1}\left|f\left(s, \tilde{\phi}_{s}+\tilde{z}_{s}\right)\right| d s\right] \\
\leq & \frac{t_{2}^{1-\alpha}-t_{1}^{1-\alpha}}{\Gamma(\alpha)} \int_{0}^{t_{2}}\left(t_{2}-s\right)^{\alpha-1} \eta(s) \Omega\left(\left\|\tilde{\phi}_{s}+\tilde{z}_{s}\right\|_{\mathcal{B}}\right) d s \\
& +\frac{t_{1}^{1-\alpha}}{\Gamma(\alpha)}\left[\int_{0}^{t_{1}}\left(\left(t_{1}-s\right)^{\alpha-1}-\left(t_{2}-s\right)^{\alpha-1}\right) \eta(s) \Omega\left(\left\|\tilde{\phi}_{s}+\tilde{z}_{s}\right\|_{\mathcal{B}}\right) d s\right. \\
& \left.+\int_{t_{1}}^{t_{2}}\left(t^{2}-s\right)^{\alpha-1} \eta(s) \Omega\left(\left\|\tilde{\phi}_{s}+\tilde{z}_{s}\right\|_{\mathcal{B}}\right) d s\right] \\
\leq & \frac{\left(t_{2}^{1-\alpha}-t_{1}^{1-\alpha}\right) \Omega\left(r_{0}\right)}{\Gamma(\alpha)}\left(\int_{0}^{t_{2}}\left(t_{2}-s\right)^{(\alpha-1) q} d s\right)^{1 / q}\left(\int_{0}^{t_{2}} \eta^{p}(s) d s\right)^{1 / p} \\
& +\frac{t_{1}^{1-\alpha} \Omega\left(r_{0}\right)}{\Gamma(\alpha)}\left[\left(\int_{0}^{t_{1}}\left(\left(t_{1}-s\right)^{\alpha-1}-\left(t_{2}-s\right)^{\alpha-1}\right)^{q} d s\right)^{1 / q}\left(\int_{0}^{t_{1}} \eta^{p}(s) d s\right)^{1 / p}\right. \\
& \left.+\left(\int_{t_{1}}^{t_{2}}\left(t^{2}-s\right)^{(\alpha-1) q} d s\right)^{1 / q}\left(\int_{0}^{t_{1}} \eta^{p}(s) d s\right)^{1 / p}\right] \\
\leq & \frac{\|\eta\|_{p} \Omega\left(r_{0}\right)}{r_{1} \Gamma(\alpha)}\left(b^{r_{2}}\left(t_{2}^{1-\alpha}-t_{1}^{1-\alpha}\right)+b^{1-\alpha}\left(2\left(t_{2}-t_{1}\right)^{r_{2}}+\left(t_{2}^{r_{2}}-t_{1}^{r_{2}}\right)\right)\right),
\end{aligned}
$$

where $r_{0}=K_{b} r+M_{b}\|\phi\|_{\mathcal{B}}, r_{1}=((\alpha-1) q+1)^{1 / q}$ and $r_{2}=[(\alpha-1) q+1] / q>0$. It follows that $\left|t_{2}^{1-\alpha}(P z)\left(t_{2}\right)-t_{1}^{1-\alpha}(P z)\left(t_{1}\right)\right| \rightarrow 0$ as $t_{2}-t_{1} \rightarrow 0$, and the convergence is independent of $z \in B_{r}$, which implies that the set $P B_{r}$ is equicontinuous.

Now we have proved that $P$ maps bounded subsets into bounded and equicontinuous subsets in $W$. By the Arzelá-Ascoli theorem, we conclude that $P$ is a completely continuous operator.

To apply Schauder's fixed point theorem, we need to verify that there exists a closed convex bounded subset in $B \subset W$ such that $P B \subset B$. To this end, we derive from inequality (3.5) that there exists a constant $\tilde{r}>0$ such that

$$
\frac{K_{b} b^{2}\|\eta\|_{p}}{r_{1} \Gamma(\alpha)} \Omega\left(K_{b} \tilde{r}+M_{b}\|\phi\|_{\mathcal{B}}\right)+|\phi(0)|<\tilde{r} .
$$

Define $B=\left\{z \in W ;\|z\|_{W} \leq \tilde{r}\right\}$, then $B$ is closed, convex and bounded in $W$. Then, for every $z \in B$ and $t \in(0, b]$, we have

$$
\begin{aligned}
\left|t^{1-\alpha}(P z)(t)\right| & \leq \frac{t^{1-\alpha}}{\Gamma(\alpha)} \int_{0}^{t}(t-s)^{\alpha-1}\left|f\left(s, \tilde{\phi}_{s}+\tilde{z}_{s}\right)\right| d s+|\phi(0)| \\
& \leq \frac{b^{1-\alpha}}{\Gamma(\alpha)} \int_{0}^{t}(t-s)^{\alpha-1} \eta(s) \Omega\left(\left\|\tilde{\phi}_{s}+\tilde{z}_{s}\right\|_{\mathcal{B}}\right) d s+|\phi(0)|
\end{aligned}
$$




$$
\begin{aligned}
& \leq \frac{b^{1-\alpha}}{\Gamma(\alpha)}\left(\int_{0}^{t}(t-s)^{(\alpha-1) q} d s\right)^{1 / q}\|\eta\|_{p} \Omega\left(K_{b} \tilde{r}+M_{b}\|\phi\|_{\mathcal{B}}\right)+|\phi(0)| \\
& \leq \frac{K_{b} b^{2}\|\eta\|_{p}}{r_{1} \Gamma(\alpha)} \Omega\left(K_{b} \tilde{r}+M_{b}\|\phi\|_{\mathcal{B}}\right)+|\phi(0)| \\
& \leq \tilde{r} .
\end{aligned}
$$

It follows that $\|P z\|_{W} \leq \tilde{r}$ for all $z \in B$, and hence $P B \subset B$.

An application of Schauder's fixed point theorem shows that there exists at least a fixed point $z$ of $P$ in $W$. Then $y=z+\tilde{\phi}$ is the solution to (1.9)-(1.10), and the proof is completed.

\section{Dependence on initial data}

In this section, we investigate the influence of a perturbation of initial data to the solutions. We first look at the dependence of solutions on initial values. For this purpose, we denote by $y(\phi, \cdot)$ the solution to equation (1.9) with initial condition (1.10), and by $y(\psi, \cdot)$ the solution to (1.9) with the initial condition

$$
\tilde{y}_{0}=\psi \in \mathcal{B}
$$

We will prove that the solution mapping is Lipschitz continuous.

Theorem 4.1 Let the assumptions of Theorem 3.2 hold. If $L K_{b} b / \Gamma(1+\alpha)<1$, then there exists a constant $\tilde{L}$ such that

$$
\|y(\phi, \cdot)-y(\psi, \cdot)\|_{C_{1-\alpha}} \leq \tilde{L}\|\phi-\psi\|_{\mathcal{B}}
$$

for every $\phi, \psi \in \mathcal{B}$.

Proof From Theorem 3.2 we know that for every $\phi, \psi \in \mathcal{B}$, equation (1.9) has solutions $y(\phi, \cdot)$ and $y(\psi, \cdot)$ on $(-\infty, b]$, respectively. Further, there are $z_{1}, z_{2} \in C_{1-\alpha}((0, b])$ such that $y(\phi, \cdot)=z_{1}+\tilde{\phi}$ and $y(\psi, \cdot)=z_{2}+\tilde{\psi}$, satisfying

$$
\begin{aligned}
& z_{1}(t)=\frac{1}{\Gamma(\alpha)} \int_{0}^{t}(t-s)^{\alpha-1} f\left(s, \tilde{\phi}_{s}+\tilde{z}_{1 s}\right) d s+\phi(0) t^{\alpha-1}, \quad t \in(0, b] \\
& z_{2}(t)=\frac{1}{\Gamma(\alpha)} \int_{0}^{t}(t-s)^{\alpha-1} f\left(s, \tilde{\psi}_{s}+\tilde{z}_{2 s}\right) d s+\psi(0) t^{\alpha-1}, \quad t \in(0, b]
\end{aligned}
$$

and $y(\phi, t)=z_{1}(t), y(\psi, t)=z_{2}(t)$ for $t \in(0, b]$. Then, by $\left(\mathrm{H}_{2}\right)$ and axiom (A1) (in Definition 2.4), for $t \in(0, b]$ we have

$$
\begin{aligned}
& \left|t^{1-\alpha} y(\phi, t)-t^{1-\alpha} y(\psi, t)\right| \\
& \quad=\left|t^{1-\alpha} z_{1}(t)-t^{1-\alpha} z_{2}(t)\right| \\
& \quad \leq \frac{t^{1-\alpha}}{\Gamma(\alpha)} \int_{0}^{t}(t-s)^{\alpha-1}\left|f\left(s, \tilde{\phi}_{s}+\tilde{z}_{1 s}\right)-f\left(s, \tilde{\psi}_{s}+\tilde{z}_{2 s}\right)\right| d s+|\phi(0)-\psi(0)| \\
& \quad \leq \frac{L t^{1-\alpha}}{\Gamma(\alpha)} \int_{0}^{t}(t-s)^{\alpha-1}\left(\left\|\tilde{z}_{1 s}-\tilde{z}_{2 s}\right\|_{\mathcal{B}}+\left\|\tilde{\phi}_{s}-\tilde{\psi}_{s}\right\|_{\mathcal{B}}\right) d s+|\phi(0)-\psi(0)|
\end{aligned}
$$




$$
\begin{aligned}
\leq & \frac{L t^{1-\alpha}}{\Gamma(\alpha)}\left[\int_{0}^{t}(t-s)^{\alpha-1} K(s) \sup _{0 \leq \tau \leq s}\left|\tilde{z}_{1}(\tau)-\tilde{z}_{2}(\tau)\right| d s\right. \\
& \left.+\int_{0}^{t}(t-s)^{\alpha-1} M(s)\left\|\tilde{\phi}_{0}-\tilde{\psi}_{0}\right\|_{\mathcal{B}} d s\right]+H\|\phi-\psi\|_{\mathcal{B}} \\
\leq & \frac{L t^{1-\alpha}}{\Gamma(\alpha)}\left[\int_{0}^{t}(t-s)^{\alpha-1} d s K_{b}\left\|z_{1}-z_{2}\right\|_{C_{1-\alpha}}\right. \\
& \left.+\int_{0}^{t}(t-s)^{\alpha-1} d s M_{b}\|\phi-\psi\|_{\mathcal{B}}\right]+H\|\phi-\psi\|_{\mathcal{B}} \\
\leq & \frac{L K_{b} b}{\Gamma(1+\alpha)}\left\|z_{1}-z_{2}\right\|_{C_{1-\alpha}}+\left(\frac{L M_{b} b}{\Gamma(1+\alpha)}+H\right)\|\phi-\psi\|_{\mathcal{B}},
\end{aligned}
$$

and hence

$$
\begin{aligned}
& \|y(\phi, \cdot)-y(\psi, \cdot)\|_{C_{1-\alpha}} \\
& \quad \leq \frac{L K_{b} b}{\Gamma(1+\alpha)}\left\|z_{1}-z_{2}\right\|_{C_{1-\alpha}}+\left(\frac{L M_{b} b}{\Gamma(1+\alpha)}+H\right)\|\phi-\psi\|_{\mathcal{B}} \\
& \quad=\frac{L K_{b} b}{\Gamma(1+\alpha)}\|y(\phi, \cdot)-y(\psi, \cdot)\|_{C_{1-\alpha}}+\left(\frac{L M_{b} b}{\Gamma(1+\alpha)}+H\right)\|\phi-\psi\|_{\mathcal{B}} .
\end{aligned}
$$

Since $L K_{b} b / \Gamma(1+\alpha)<1$, let $\tilde{L}=\left(L M_{b} b+H \Gamma(1+\alpha)\right) /\left(\Gamma(1+\alpha)-L K_{b} b\right)$. Then we obtain

$$
\|y(\phi, \cdot)-y(\psi, \cdot)\|_{C_{1-\alpha}} \leq \tilde{L}\|\phi-\psi\|_{\mathcal{B}}
$$

as desired, which completes the proof.

Next we investigate the influence of changes in the given function on the right-hand side of the differential equation. Now we denote by $y(f, \cdot)$ the solution to the differential equation (1.9) with initial condition (1.10) and by $y(\tilde{f}, \cdot)$ the solution to the differential equation

$$
D_{0}^{\alpha} y(t)=\tilde{f}\left(t, \tilde{y}_{t}\right), \quad t \in(0, b]
$$

with initial condition (1.10).

Theorem 4.2 Let $f$ and $\tilde{f}$ fulfill hypotheses $\left(\mathrm{H}_{1}\right)$ and $\left(\mathrm{H}_{2}\right)$. If $L K_{b} b / \Gamma(1+\alpha)<1$, then there exists a constant $\tilde{K}$ such that

$$
\|y(f, \cdot)-y(\tilde{f}, \cdot)\|_{C_{1-\alpha}} \leq \tilde{K} \sup _{(t, v) \in(0, b] \times \mathcal{B}}|f(t, v)-\tilde{f}(t, v)| .
$$

Proof The existence of solutions can be ensured by Theorem 3.2. Let $z_{1}, z_{2} \in C_{1-\alpha}((0, b])$ be such that $y(f, \cdot)=z_{1}+\tilde{\phi}$ and $y(\tilde{f}, \cdot)=z_{2}+\tilde{\phi}$. Then $z_{1}$ and $z_{2}$ satisfy

$$
z_{1}(t)=\frac{1}{\Gamma(\alpha)} \int_{0}^{t}(t-s)^{\alpha-1} f\left(s, \tilde{\phi}_{s}+\tilde{z}_{1 s}\right) d s+\phi(0) t^{\alpha-1}, \quad t \in(0, b]
$$

and

$$
z_{2}(t)=\frac{1}{\Gamma(\alpha)} \int_{0}^{t}(t-s)^{\alpha-1} \tilde{f}\left(s, \tilde{\phi}_{s}+\tilde{z}_{2 s}\right) d s+\phi(0) t^{\alpha-1}, \quad t \in(0, b] .
$$


For $t \in(0, b]$, we have

$$
\begin{aligned}
\mid t^{1-\alpha} & y(f, t)-t^{1-\alpha} y(\tilde{f}, t) \mid \\
= & \left|t^{1-\alpha} z_{1}(t)-t^{1-\alpha} z_{2}(t)\right| \\
\leq & \frac{t^{1-\alpha}}{\Gamma(\alpha)} \int_{0}^{t}(t-s)^{\alpha-1}\left|f\left(s, \tilde{\phi}_{s}+\tilde{z}_{1 s}\right)-\tilde{f}\left(s, \tilde{\phi}_{s}+\tilde{z}_{2 s}\right)\right| d s \\
\leq & \frac{t^{1-\alpha}}{\Gamma(\alpha)}\left[\int_{0}^{t}(t-s)^{\alpha-1}\left|f\left(s, \tilde{\phi}_{s}+\tilde{z}_{1 s}\right)-f\left(s, \tilde{\phi}_{s}+\tilde{z}_{2 s}\right)\right| d s\right. \\
& \left.+\int_{0}^{t}(t-s)^{\alpha-1}\left|f\left(s, \tilde{\phi}_{s}+\tilde{z}_{2 s}\right)-\tilde{f}\left(s, \tilde{\phi}_{s}+\tilde{z}_{2 s}\right)\right| d s\right] \\
\leq & \frac{L K_{b} b}{\Gamma(1+\alpha)}\left\|z_{1}-z_{2}\right\|_{C_{1-\alpha}}+\frac{L b}{\Gamma(1+\alpha)} \sup _{(t, v) \in(0, b] \times \mathcal{B}}|f(t, v)-\tilde{f}(t, v)| .
\end{aligned}
$$

Therefore,

$$
\begin{aligned}
& \|y(f, \cdot)-y(\tilde{f}, \cdot)\|_{C_{1-\alpha}} \\
& \quad \leq \frac{L K_{b} b}{\Gamma(1+\alpha)}\left\|z_{1}-z_{2}\right\|_{C_{1-\alpha}}+\frac{L b}{\Gamma(1+\alpha)} \sup _{(t, v) \in(0, b] \times \mathcal{B}}|f(t, v)-\tilde{f}(t, v)| \\
& \quad=\frac{L K_{b} b}{\Gamma(1+\alpha)}\|y(f, \cdot)-y(\tilde{f}, \cdot)\|_{C_{1-\alpha}}+\frac{L b}{\Gamma(1+\alpha)} \sup _{(t, v) \in(0, b] \times \mathcal{B}}|f(t, v)-\tilde{f}(t, v)| .
\end{aligned}
$$

Take $\tilde{K}=L b /\left(\Gamma(1+\alpha)-L K_{b} b\right)$, we get the required result.

\section{An example}

In this section we give an example to illustrate our main results. Let $B_{\gamma}=\{y \in C((-\infty, 0]$; $\mathbf{R}) ; \lim _{\theta \rightarrow-\infty} e^{\gamma \theta} y(\theta)$ exist in $\left.\mathbf{R}\right\}$, where $\gamma>0$ is a constant. Then $B_{\gamma}$ satisfies axioms (A1), (A2) and (B) in Definition 2.4 with $K(t)=M(t)=1$ and $H=1$ [13]. For any $\phi \in B_{\gamma}$, consider the weighted fractional functional differential equation

$$
D^{\alpha} y(t)=\lambda\left[t^{1-\alpha} y(t-b)\right]^{\beta}+g(t), \quad t \in(0, b],
$$

with infinite delay

$$
y(t)=\phi(t), \quad t \in(-\infty, 0],
$$

where $g \in C([0,1]), \lambda \in \mathbf{R}$ and $\beta>0$ are constants. Let $f(t, x)=\lambda x^{\beta}+g(t)$. If $\beta \geq 1$, then it is easily seen that $f$ satisfies the Lipschitz condition with respect to $x$ on any bounded interval. So, by Theorem 3.2, problem (5.1)-(5.2) has a unique solution on $(-\infty, b]$. If $0<\beta<1$, then $f$ does not satisfy the Lipschitz condition with respect to $x$ in some neighborhoods of 0 . However, in this case, we can define $\eta(t)=\sup \{g(t) ; t \in[0,1]\}, \Omega(r)=1$ for $r \in[0,1]$, and $\Omega(r)=r^{\rho}$ for $r \geq 1$ and constant $\rho$ with $\beta<\rho<1$. Then hypothesis $\left(\mathrm{H}_{3}\right)$ holds. Also, since $\limsup _{r \rightarrow \infty} \Omega(r) / r=0$, condition (3.5) is satisfied. Hence, by Theorem 3.3, problem (5.1)-(5.2) has at least a solution on $(-\infty, b]$. 
Remark 5.1 In [2], the authors studied the weighted fractional Cauchy problem

$$
\left(D_{a+}^{\alpha} y\right)(t)=\lambda(t-a)^{\beta}[y(t)]^{m}+b(t-a)^{\nu},
$$

where $\alpha>0, t>a$ and $m>0$ with initial conditions

$$
\left(D_{a+}^{\alpha-k} y\right)(a+)=b_{k} \quad\left(b_{k} \in \mathbf{R}, k=1,2, \ldots, n ; n=[\alpha]+1\right) .
$$

The nonlinear case $(m \neq 1)$ is discussed in Example 3.4 on $p .181$, and the linear case $(m=1)$ in Examples 4.2-4.3 on pp.225-227. For the homogeneous case, the explicit solutions are constructed by using generalized Mittag-Leffler functions. However, in general, we cannot expect to find explicit solutions for delayed fractional differential equations, even for linear and homogeneous cases.

\section{Competing interests}

The author declares that he has no competing interests.

\section{Acknowledgements}

This research was done while the author visited the University of Southern Mississippi, which was supported financially by Jiangsu Government Scholarship for Overseas Studies (JS-2011-159). This work is supported by the National Natural Science Foundation of China (11271316 and 11201410) and the Natural Science Foundation of Jiangsu Province (BK2012260 and BK20141271).

\section{Received: 2 May 2014 Accepted: 1 July 2014 Published: 22 Jul 2014}

\section{References}

1. Diethelm, K: The Analysis of Fractional Differential Equations. Lecture Notes in Mathematics, vol. 2004. Springer, Berlin (2010)

2. Kilbas, AA, Srivastava, HM, Trujillo, JJ: Theory and Applications of Fractional Differential Equations. North-Holland Math. Stud., vol. 204. Elsevier, Amsterdam (2006)

3. Delbosco, D, Rodino, L: Existence and uniqueness for a nonlinear fractional differential equation. J. Math. Anal. Appl. 204, 609-625 (1996)

4. Yang, $X$, Liu, Y: Picard iterative processes for initial value problems of singular fractional differential equations. Adv. Differ. Equ. 2014, Article ID 102 (2014)

5. Benchohra, M, Henderson, J, Ntouyas, SK, Ouahab, A: Existence results for fractional order functional differential equations with infinite delay. J. Math. Anal. Appl. 338, 1340-1350 (2008)

6. Henderson, J, Ouahab, A: Fractional functional differential inclusions with finite delay. Nonlinear Anal. 70, 2091-2105 (2009)

7. Zhang, X: Some results of linear fractional order time-delay system. Appl. Math. Comput. 197, 407-411 (2008)

8. Lakshmikantham, V: Theory of fractional functional differential equations. Nonlinear Anal. 69, 3337-3343 (2008)

9. Lakshmikantham, V, Devi, JV: Theory of fractional differential equations in Banach space. Eur. J. Pure Appl. Math. 1(1), 38-45 (2008)

10. Darwish, MA, Ntouyas, SK: On initial and boundary value problem for fractional order mixed type functional differential inclusions. Comput. Math. Appl. 59, 1253-1265 (2010)

11. Agarwal, R, Ntouyas, SN, Ahmad, B, Alhothuali, MS: Existence of solutions for integro-differential equations of fractional order with nonlocal three-point fractional boundary conditions. Adv. Differ. Equ. 2013, Article ID 128 (2013)

12. Dong, $Q, W u, G, L i, ~ J:$ Boundary value problem for a class of fractional differential equations in Banach spaces. Pure Appl. Math. 29(1), 1-10 (2003)

13. Hino, Y, Murakami, S, Naito, T: Functional Differential Equations with Infinite Delay. Lecture Notes in Mathematics, vol. 1473. Springer, Berlin (1991)

14. Hale, JK, Kato, J: Phase space for retarded equations with infinite delay. Funkc. Ekvacioj 21, 11-41 (1978)

10.1186/1687-1847-2014-190

Cite this article as: Dong: Existence and continuous dependence for weighted fractional differential equations with infinite delay. Advances in Difference Equations 2014, 2014:190 